Research Paper

\title{
Modified three-level techniques of retroperitoneal laparoscopic procedures to treat adrenal lesions for patients with $\mathrm{BMI} \geq 25 \mathrm{Kg} / \mathrm{m}^{2}$
}

\author{
Minxiong $\mathrm{Hu}^{1}$, Zesong Yang${ }^{2}$, Yuandong $\mathrm{Chen}^{3}$, Guangbing Chen" ${ }^{4}$ Zhensheng $\mathrm{Chen}^{5}$, Tao Li ${ }^{1}$, Qingguo \\ Zhu1 ${ }^{1}$, Yongbao Wei ${ }^{{ }^{\circledR \#}}$ and Liefu Ye ${ }^{{ }^{\bowtie \#}}$ \\ 1. Shengli Clinical Medical College of Fujian Medical University and Department of Urology, Fujian Provincial Hospital, Fuzhou 350001, China. \\ 2. Department of Urology, Fujian Provincial Hospital South Branch, Fuzhou 350028, China. \\ 3. Fujian Medical University, Fuzhou 350004, China. \\ 4. Department of Urology, Min Dong Hospital of Ningde City Ningde, Fujian Province, China. \\ 5. Department of Urology, Fuding Hospital Affiliated to Fujian University of Traditional Chinese Medicine, Fuding City, Fujian Province, China. \\ \#Co-corresponding authors with equal contributions to this work. \\ $\square$ Corresponding author: Yongbao Wei, MD (E-mail: weiyb@fjmu.edu.cn; Tel.: +8615859192139) or Liefu Ye, PhD (E-mail: liefu2001@sina.com; Tel.: \\ +8613696885658), Shengli Clinical Medical College of Fujian Medical University and Department of Urology, Fujian Provincial Hospital, Fuzhou 350001, China. \\ (C) The author(s). This is an open access article distributed under the terms of the Creative Commons Attribution License (https://creativecommons.org/licenses/by/4.0/). \\ See http://ivyspring.com/terms for full terms and conditions.
}

Received: 2020.06.16; Accepted: 2020.10.19; Published: 2020.10.22

\begin{abstract}
Objective: To evaluate the modified Zhang's 'three-level' technique of retroperitoneal laparoscopic adrenalectomy (RLA) to treat adrenal lesions for patients with BMI of $25-30 \mathrm{Kg} / \mathrm{m}^{2}$.

Methods: A retrospective analysis was performed in all patients with BMI of $25-30 \mathrm{Kg} / \mathrm{m}^{2}$ in our hospital from January 2014 to December 2019. Those who underwent laparoscopic adrenal surgery were divided into two groups on the basis of the technique used: the Zhang's technique (the ZT group) and the modified technique (the MT group).

Results: Herein, 170 operations were included (ZT, 91 patients; MT, 79 patients). RLA was successfully performed in all of them. Compared with the ZT group patients, the MT group patients showed shorter operation time $(p=0.007)$, lesser intraoperative blood loss $(p=0.023)$, shorter operation time, earlier postoperative diet recovery $(p<0.001)$, shorter postoperative drainage time $(p<0.001)$ and shorter postoperative hospitalization period $(p=0.001)$. It was also worth noting that the unplanned total adrenalectomy rate was significantly less in the MT group than in the ZT group ( $0 \%$ vs. $10.8 \%, p=0.020)$. There was no significant difference in the complications between the two groups $(3.3 \%$ vs. $2.5 \%, p=$ 0.567).

Conclusions: We found that MT was a beneficial retroperitoneal laparoscopic treatment for adrenal lesions in patients who had a BMl of $25-30 \mathrm{Kg} / \mathrm{m}^{2}$. It may provide a reference for the treatment of adrenal surgical diseases in such patients.
\end{abstract}

Key words: adrenal gland, retroperitoneal laparoscopy, BMI, fat

\section{Introduction}

Over the past three decades, laparoscopic adrenalectomy has replaced open adrenalectomy and become the gold standard for surgical treatment of most adrenal diseases [1]. Transperitoneal laparoscopy is one of the most widely used adrenal approaches [2] because it allows for the best comprehensive observation of the adrenal region and its surrounding tissues. Gagner was the first to perform retroperitoneal laparoscopic adrenalectomy (RLA) in 1992 [3]. Both retroperitoneal and transabdominal laparoscopic treatments of benign adrenal tumours are now considered safe and effective, with similar outcomes [4]. Even RLA has lesser postoperative pain and shorter hospitalization 
periods than the transabdominal approach [5]. The retroperitoneal approach has its unique advantages, making it a valuable alternative method to treat the adrenal gland lesions [6]. RLA has recently become more popular [7] and its advantage is that it allows direct access to the retroperitoneal cavity despite the small working space. Therefore, it avoids the abdominal cavity and limits the risk of vascular and other visceral injuries [8], thus resulting in less postoperative pain and shorter hospitalizations [5]. In 2007, Zhang et al. reported their 'three-level' method of RLA, which involved programmed and simplified procedures of RLA [9]. The learning curve of this method seems shorter [10], and it has become a widely accepted approach to treat adrenal surgical diseases in mainland China in the last decade.

During our clinical practice, we found the 'three-level' RLA suitable for most patients. However, it seems not an effective enough method to treat overweight and obese patients as their relatively thick peri-renal fat is makes the retroperitoneal space relatively narrow. Some patients have peri-renal fat adhesion, which makes it more difficult when separating the upper pole of the kidney from the peri-renal fat. Even after an effort-intensive process of separation, the adrenal glands are still invisible because of the upper pole fat. Thus, we apply this method of changing the third level approach to make the procedure more convenient to perform for overweight and obese patients.

\section{Materials and Methods}

\section{Patients}

We retrospectively included patients who had undergone adrenal surgery in our department from January 2014 to December 2019. Patients who met the following criteria were included: (a) RLA was performed for the patients with adrenal tumours or lesions; (b) patients with $\mathrm{BMI} \geq 25 \mathrm{Kg} / \mathrm{m}^{2}$; (c) the surgical procedure was Zhang's standard three-level method or the modified one; (d) patients underwent CT or MRI scan of the abdomen before operation; (e) the patients accepted RLA by the same skilled surgeon in our department.

The exclusion criteria were that patients did not meet the above conditions at the same time, the patients were not willing to participate in this study or the data available was insufficient for analysis.

We analysed the effectives and peri-operative safety of our modified surgical approach compared with Zhang' s standard 'three-level' method for overweight and obese patients with BMI $\geq 25 \mathrm{Kg} / \mathrm{m}^{2}$ [11].

The study was approved from by the Ethics
Committee of our hospital. Written informed consents were obtained from the guardians of these patients.

\section{Surgical approach}

Key points of Zhang's standard 'three-level' technique (ZT)

Preoperative preparations were made according to the patient's preoperative diagnoses. This technique was performed as per the protocol of Zhang et al. [9]. Briefly, first, the retroperitoneal space was prepared. Then, three trocars were placed as working channels. The pressure of $\mathrm{CO}_{2}$ pneumoperitoneum was maintained at $11 \mathrm{mmHg}(1 \mathrm{mmHg}=0.133 \mathrm{kPa})$. The extra-peritoneal fat was removed and Gerota's fascia was separated. Then, we performed the first separation of the relative avascular space between the adrenal fat capsule and the anterior Gerota's fascia (Fig.1A). Then, in the second separation level, the relatively avascular space was between the dorsal side of the adrenal fat capsule and the psoas muscle (Fig.1B). The third separation level was located between the surface of the upper adrenal capsule and the peri-renal fat, dissociating it with the lower adrenal pole (Fig. 1C).

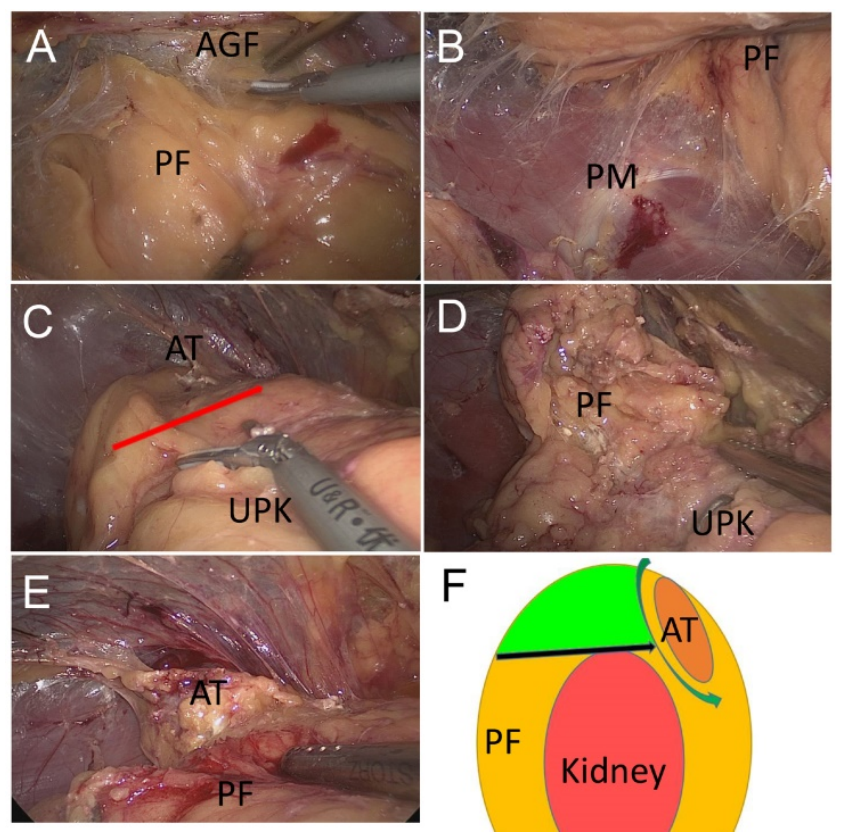

Figure 1. Surgical approach of 'three-level' and modified 'three-level' methods. (A) The first level: between anterior Gerota's fascia (AGF) and perinephric fat (PF) that located at the superomedial side of the kidney. (B) The second level: between lateral perinephric fat (PF) and psoas muscle (PM). (C) The third level (shown by the arrow): between the bottom of adrenal tumour (AT) and upper pole of the kidney (UPK). (D) When adherent or large perinephric fat (PF) exists, the third level is not easy to obtain and the adrenal tumour is not easy to access. (E) The modified third level: pull the perinephric fat (PF) away, and the adrenal tumour (AT) is visible clearly. (F) A diagram of the modified 'three-level' approach. The green area is the redundant perinephric fat (PF) in this technique. This fat can be used as a pull position to give enough operation view to discover the adrenal tumour (AT). 
Key points of modified three-level Technique (MT)

During our clinical practice, we have identified that when adherent or large peri-nephric fat exists, the traditional third level intervention is not easy to perform and the adrenal tumour is not easy to access (Fig. 1D). Thus, we modified the third level of this approach. The operation method of our modified third level was essentially the same as Zhang's standard three-level method, except that the separation was improved at the third separation level: the peripheral renal fat was dissected from the dorsal side of the upper pole to the adrenal gland. After reaching the adrenal gland, the adrenal gland and the adenoma were exposed along the edge of the adrenal gland (Fig.1E). The auxiliary hand could then pull the peri-renal fat outwards to create a large enough operation space at the upper pole of the kidney (Fig. $1 F)$.

\section{Statistical analysis}

Statistical software SPSS 24.0 was used for analysis. The independent sample $t$ test or chi-square test was used for comparison. A $p$ value of $<0.05$ was considered statistical significance.

\section{Results}

\section{General demographic data}

A total of 170 cases were included in this study. All patients successfully underwent endoscopic surgery, and none was converted to open surgery. There were 91 cases in the ZT group and 79 cases in the MT group. The ZT group had 45 men and 46 women, with an average BMI of $27.68 \mathrm{Kg} / \mathrm{m}^{2} \pm 2.94$ $\mathrm{Kg} / \mathrm{m}^{2}$ and an average tumour diameter of $4.21 \mathrm{~cm} \pm$ $1.29 \mathrm{~cm}$. The MT group had 44 men and 35 women, with an average BMI of $27.45 \mathrm{Kg} / \mathrm{m}^{2} \pm 1.89 \mathrm{Kg} / \mathrm{m}^{2}$ and an average tumour diameter of $4.27 \mathrm{~cm} \pm 1.35 \mathrm{~cm}$. In the MT group, the lesions were located on the left in 44 cases and on the right in 35 cases. There was no statistically significant difference between the two groups in terms of gender, BMI, tumour diameter, tumour type and left- and right-side comparison (all $p$ $>0.05$ ). Patients in the ZT group were marginally older than those in the MT group [ZT, $55.10 \pm 10.89$ years vs. MT, $51.52 \pm 11.93$ years $p<0.05]$ (Table 1 ).

\section{Comparison of peri-operative period between the two groups}

The ZT group planned reserving 74 cases adrenal glands $(82.2 \%)$ and 17 cases $(17.8 \%)$ of planned adrenalectomy, but finally, there were nine cases $(12.2 \%)$ accepted as unplanned adrenalectomy. The MT group planned reserving 61 cases of adrenal glands $(77.2 \%)$ and 18 cases of planned total adrenalectomy (22.8\%); no unplanned cases $(0 \%)$ resulted in total adrenalectomy. Obviously, the proportion unplanned adrenalectomy in the MT group was lower $(p<0.05)$ (Table 2$)$. The average operation time of the ZT group was $115.89 \pm 34.94 \mathrm{~min}$ and blood loss were $45.22 \pm 66.07 \mathrm{ml}$; the average postoperative recovery time was $1.63 \pm 0.95$ days. The drainage tube extraction time in the $\mathrm{ZT}$ group was $4.79 \pm 2.21$ days; its average postoperative hospitalisation period was $6.78 \pm 3.74$ days. The average operation time in the MT group was $102.49 \pm$ $28.20 \mathrm{~min}$ and blood loss were $28.42 \pm 20.06 \mathrm{ml}$; postoperative recovery time was $1.15 \pm 0.48$ days. The postoperative drainage tube extraction time was 3.47 \pm 1.52 days, and the postoperative hospitalisation period was $4.78 \pm 3.73$ days. On comparing the two groups, it was found that compared with the ZT group, the MT group had a shorter operation time, lesser blood loss, shorter postoperative diet recovery time, earlier postoperative drainage tube removal and shorter postoperative hospitalisation period (all $p<$ $0.05)$. The incidences of complications in both groups were very low $(p>0.05)$ (Table 3$)$, including three cases $(3.3 \%$ ) in the ZT group (two cases of subcutaneous emphysema and one case of incisional hernia) and two cases (2.5\%) in the MT group (one case of subcutaneous emphysema and one case of chylic leakage).

Table 1. General demographic data

\begin{tabular}{llll}
\hline & ZT & MT & $P$ value \\
\hline Gender (n) & 45 & 44 & 0.374 \\
Male & 46 & 35 & \\
Female & $55.10 \pm 10.89$ & $51.52 \pm 11.93$ & 0.042 \\
Age (years) & $27.68 \pm 2.94$ & $27.45 \pm 1.89$ & 0.548 \\
BMI (Kg/m²) & & & \\
Tumour location (n) & 54 & 44 & 0.683 \\
Left & 37 & 35 & \\
Right & $4.21 \pm 1.29$ & $4.27 \pm 1.35$ & 0.733 \\
Tumour size (cm) & & & \\
Tumour type (n) & 52 & 42 & \\
Incidental tumour & 2 & 5 & 1.000 \\
Pheochromocytoma & 37 & 32 & \\
Others & & \\
\hline
\end{tabular}

ZT, Zhang's technique; MT, modified technique.

Table 2. The rates of unplanned adrenalectomy between the two groups

\begin{tabular}{lllll}
\hline & $\begin{array}{l}\text { Planned } \\
\text { adrenalectomy }\end{array}$ & $\begin{array}{l}\text { Planed } \\
\text { adrenal } \\
\text { sparing }\end{array}$ & $\begin{array}{l}\text { Unplanned } \\
\text { adrenalectomy }\end{array}$ & $\begin{array}{l}\text { The rate of } \\
\text { unplanned } \\
\text { adrenalectomy }(\%)\end{array}$ \\
\hline ZT & 17 & 74 & 9 & $10.8 \%$ \\
MT & 18 & 61 & 0 & 0 \\
$P$ value & & 0.020 & & \\
\hline
\end{tabular}

ZT, Zhang's technique; MT, modified technique. 
Table 3. Comparison of perioperative period between the two groups

\begin{tabular}{llll}
\hline & ZT & MT & $P$ value \\
\hline Operation time (min) & $115.89 \pm 34.94$ & $102.49 \pm 28.20$ & 0.007 \\
Blood loss (ml) & $45.22 \pm 66.07$ & $28.42 \pm 20.06$ & 0.023 \\
Diet recovery (days) & $1.63 \pm 0.95$ & $1.15 \pm 0.48$ & $<0.001$ \\
Drainage (days) & $4.79 \pm 2.21$ & $3.47 \pm 1.52$ & $<0.001$ \\
Postoperative hospitalisations (days) & $6.78 \pm 3.74$ & $4.78 \pm 3.73$ & 0.001 \\
Complications (n) & $3 / 91(3.3 \%)$ & $2 / 79(2.5 \%)$ & 0.567 \\
\hline ZT, Zhang's technique; MT, modified technique. & &
\end{tabular}

ZT, Zhang's technique; MT, modified technique.

\section{Discussion}

Over the past few decades, the proportion of overweight and obese individuals has been rising globally; this health concern is already widespread in high-income countries and shows an increasing trend in low- and middle-income countries as well. The growth rate of this concern is the highest in men and boys in Western high-income countries and in women and girls in Central Asia, the Middle East and North Africa [12]. Being overweight or obese is a predictor of peri-renal fat adhesion, and a higher BMI indicates that peri-renal fat adhesion is more severe [13, 14]. BMI was positively correlated with high operation time, more severe postoperative complications and prolonged hospitalisations [15]. Fatty adhesions around the kidney increase the 'third level' [9] separation, which may damage the renal capsule, cause bleeding and poorly expose the surgical field, thus directly leading to prolonged operation time and even unplanned removal of the adrenal gland. The 'three-level' method was the basis of posterior laparoscopic adrenal surgery in China. A large number of clinical practices have found that the thickness of peri-renal fat in the general population is significantly different from that in overweight and obese people. We found that this 'three-level' method has limitations. The present study found that complete removal of the adrenal glands was not planned in $10.8 \%$ of overweight and obese patients; further, it was not planned in any patient in the MT group. This result suggests that the conventional 'three-level' method may be suitable for most patients, but it seems difficult to perform the 'three-level' method in overweight and obese patients, particularly in those with peri-renal fat adhesions. Therefore, for overweight and obese individuals, a modified surgical method may be necessary.

Several methods of posterior laparoscopic adrenal surgery currently exist, each with its advantages and disadvantages. Hu et al. searched for the central adrenal vein under the guidance of the renal pedicle or inferior vena cava by changing the position of the trocar and separated the relatively avascular space between the dorsal side of the peripheral renal fat capsule and Gerota's fascia [16]. Moreover, some researchers proposed 'single layer' posterior laparoscopy to treat benign adrenal diseases [17]. Compared with the 'three-level' method, the above two surgical methods do not dissociate the whole kidney, thus resulting in a lower degree of mobility, and the problem of the narrow retroperitoneal space remains as they cannot provide a wider surgical space. Although their operation may be successful, it may require more trocars and more extensive surgical experience. If complications such as a vascular injury occur during the operation, it may be more difficult to handle and would require greater expertise. Of course, more studies are needed to evaluate whether the modified 'three-level' technology is superior to these technologies in terms of the learning curve and technical advantages.

In our study, the age was different between these two groups $(p<0.05)$; the mean age of the ZT group individuals higher. This may be because of our small sample size. However, studies found that age was not an independent factor associated with prolonged time of surgery. Erbil et al. performed linear regression analysis using the operation time as the dependent variable, and they concluded that age was not an independent risk factor of the operation time [15]. This was consistent with our clinical experience. Although the operation time seems to be obviously related to tumour diameter, tumour location and BMI among other factors, in our study, no statistically significant differences were observed between the two groups in these parameters, and thus, we believed that our data of the two groups were still comparable, and the results of this study may still be practically significant.

The limitation of this study was that it was a retrospective study with a small sample size. In addition, some of these patients may have to undergo an unplanned transfer to the modified technique because of the peri-renal fat adhesion. However, we did not make stratified analyses for patients with different kinds of fatty adhesions as well as the adrenal lesions located at the medial branch because of the limited cases included. Furthermore, we had no enough reason to explain the MT group patients had the earlier postoperative diet recovery compared the patients accepted the Zhang's techniques, even which may be related to shorter operation time and/ or less blood lose. We will conduct further research on these concerns after we accumulate more cases, and we hope more studies especially randomised ones to further valuate this improved technique in future. 


\section{Conclusion}

To our knowledge, this is the first report on the application of the modified 'three-level' technique in the laparoscopic adrenal gland surgery for overweight and obese patients. This technique may provide a reference for the treatment of adrenal surgical diseases in these patients.

\section{Acknowledgements}

We thank all these patients treated in our department.

\section{Funding}

The Joint Funds for the innovation of science and Technology, Fujian province (2017Y9064) and high-level hospital foster grants from Fujian Provincial Hospital, Fujian province, China (2019HSJJ29) and the Natural Science Foundation of Fujian Province (2018J01258).

\section{Author contributions}

- YB Wei and LF Ye: Project development;

- YD Chen, GB Chen and ZS Chen: Data collection;

- T Li and QG Zhu: Data analysis. MX Hu and ZS Yang: Manuscript writing and editing.

\section{Availability of data and materials}

The data can be accessed from Liefu Ye (E-maill: liefu2001@sina.com).

\section{Research involving Human Participants}

The study was approved from by the Ethics Committee of Fujian Provincial Hospital. And we certify that the study was performed in accordance with the ethical standards as laid down in the 1964 Declaration of Helsinki and its later amendments or comparable ethical standards.

\section{Informed consent}

Written informed consents were obtained from patients' guardians.

\section{Competing Interests}

The authors have declared that no competing interest exists.

\section{References}

1. Raffaelli M, De Crea C, Bellantone R. Laparoscopic adrenalectomy. Gland surgery 2019;8(Suppl 1):S41-52.

2. Arezzo A, Bullano A, Cochetti G, et al. Transperitoneal versus retroperitoneal laparoscopic adrenalectomy for adrenal tumours in adults. The Cochrane database of systematic reviews 2018;12(12):D11668.

3. Gagner M, Lacroix A, Bolté E. Laparoscopic adrenalectomy in Cushing's syndrome and pheochromocytoma. The New England journal of medicine 1992;327(14):1033.

4. Chai YJ, Yu HW, Song R, Kim S, Choi JY, Lee KE. Lateral Transperitoneal Adrenalectomy Versus Posterior Retroperitoneoscopic Adrenalectomy for
Benign Adrenal Gland Disease: Randomized Controlled Trial at a Single Tertiary Medical Center. ANN SURG 2019;269(5):842-8.

5. Kozłowski T, Choromanska B, Wojskowicz P, et al. Laparoscopic adrenalectomy: lateral transperitoneal versus posterior retroperitoneal approach - prospective randomized trial. Wideochirurgia i inne techniki maloinwazyjne $=$ Videosurgery and other miniinvasive techniques 2019;14(2):160-9.

6. Munver R, Ilbeigi P. Retroperitoneal laparoscopic adrenalectomy. CURR UROL REP 2005;6(1):72-7.

7. Conzo G, Tartaglia E, Gambardella C, et al. Minimally invasive approach for adrenal lesions: Systematic review of laparoscopic versus retroperitoneoscopic adrenalectomy and assessment of risk factors for complications. International journal of surgery (London, England) 2016;28 Suppl 1:S118-23.

8. Klap J, Butow Z, Champy CM, et al. 1,000 Retroperitoneoscopic Procedures of the Upper Urinary Tract: Analysis of Complications. UROL INT 2019;102(4):406-12.

9. Zhang $\mathrm{X}, \mathrm{Fu} \mathrm{B}$, Lang $\mathrm{B}$, et al. Technique of anatomical retroperitoneoscopic adrenalectomy with report of 800 cases. J Urol 2007;177(4):1254-7.

10. Zhang X, Wang B, Ma X, et al. Laparoscopic adrenalectomy for beginners without open counterpart experience: initial results under staged training. UROLOGY 2009;73(5):1061-5.

11. World Health Organization. Obesity and Overweight. Geneva, Switzerland: World Health Organization. 2018; who.int/mediacentre/factsheets/ fs311/en/.

12. Sung H, Siegel RL, Torre LA, et al. Global patterns in excess body weight and the associated cancer burden. CA: a cancer journal for clinicians 2019;69(2):88-112.

13. Davidiuk AJ, Parker AS, Thomas CS, et al. Mayo adhesive probability score: an accurate image-based scoring system to predict adherent perinephric fat in partial nephrectomy. EUR UROL 2014;66(6):1165-71.

14. Khene Z, Peyronnet B, Mathieu R, Fardoun T, Verhoest G, Bensalah K. Analysis of the impact of adherent perirenal fat on peri-operative outcomes of robotic partial nephrectomy. WORLD J UROL 2015;33(11):1801-6.

15. Erbil Y, Barbaros U, Sari S, Agcaoglu O, Salmaslioglu A, Ozarmagan S. The effect of retroperitoneal fat mass on surgical outcomes in patients performing laparoscopic adrenalectomy: the effect of fat tissue in adrenalectomy. SURG INNOV 2010;17(2):114-9.

16. Hu D, Wang $X$, Liu T. Comparison of Modified Retroperitoneal Adrenalectomy With the Gold Standard for Benign Adrenal Tumors. Surgical laparoscopy, endoscopy \& percutaneous techniques 2016;26(1):86-9.

17. Li S, Wang J, Zhang E, Gao W, Yang J, Jia Z. Single-plane retroperitoneoscopic adrenalectomy: a new operative procedure for benign adrenal disease. Sci Rep 2018;8(1):4027. 